\title{
Models and Useful Relations for Bistatic SAR Processing
}

\author{
Otmar Loffeld, Holger Nies, Valerij Peters, and Stefan Knedlik, Member, IEEE
}

\begin{abstract}
The paper derives a vectorial model for expressing transmitter and receiver trajectories for arbitrary transmitter and receiver motion (nonidentical velocity vectors) in a bistatic synthetic aperture radar constellation. The point target response is first modeled in the space-time domain and then transformed to the frequency domain by the method of stationary phase giving the point target reference spectrum. In the reference spectrum, two phasor functions can be identified, the first one resembling some quasi-monostatic contribution and the second one being a bistatic deformation phasor transforming into an elliptic arc in the spatial domain. The multiplication of the two phasors in the frequency domain transforms into a convolution like operation in the space-time domain, indicating that the bistatic dataset can be expressed as a convolution like (range and azimuth variant) mapping of a monostatic dataset. In this regard, the paper extends the solution for the constant offset case known from the geophysical literature (associated with "Rocca's smile operator") showing that this case is a specialization of the more general one considered in this work.
\end{abstract}

Index Terms-Bistatic synthetic aperture radar (SAR), flat-top hyperbola, method of stationary phase, point target reference spectrum, point target response, Rocca's smile.

\section{INTRODUCTION}

B ISTATIC synthetic aperture radar (SAR) systems [1]-[3] as opposed to monostatic SAR constellations offer some degrees of freedom in choosing transmitter (illuminator) and (passive) receiver motion trajectories. The increased flexibility in designing bistatic SAR missions, however, is paid for by an increased complexity with respect to the processing of bistatic SAR raw data to SAR images. While in the monostatic SAR case, the geometry is readily modeled, and based on the models, point target response functions are evaluated and carried to the frequency domain by stationary phase techniques; the situation, however, is slightly more complex in the bistatic case. The outcoming range and phase history of any point target consists of the individual range history contributions of transmitter and receiver, where these contributions are coupled by the relative motion between transmitter (illuminator) and receiver. Furthermore, the classical monostatic correspondence between azimuth frequency and azimuth time being closely related to a certain point on the orbit is broken up, since two motion trajectories must be considered.

Manuscript received August 18, 2003; revised June 16, 2004.

The authors are with the Center for Sensorsystems (ZESS), University of Siegen, 57068 Siegen, Germany (e-mail: Loffeld@zess.uni-siegen.de; Nies@zess.uni-siegen.de; Peters@zess.uni-siegen.de; Knedlik@zess.unisiegen.de).

Digital Object Identifier 10.1109/TGRS.2004.835295

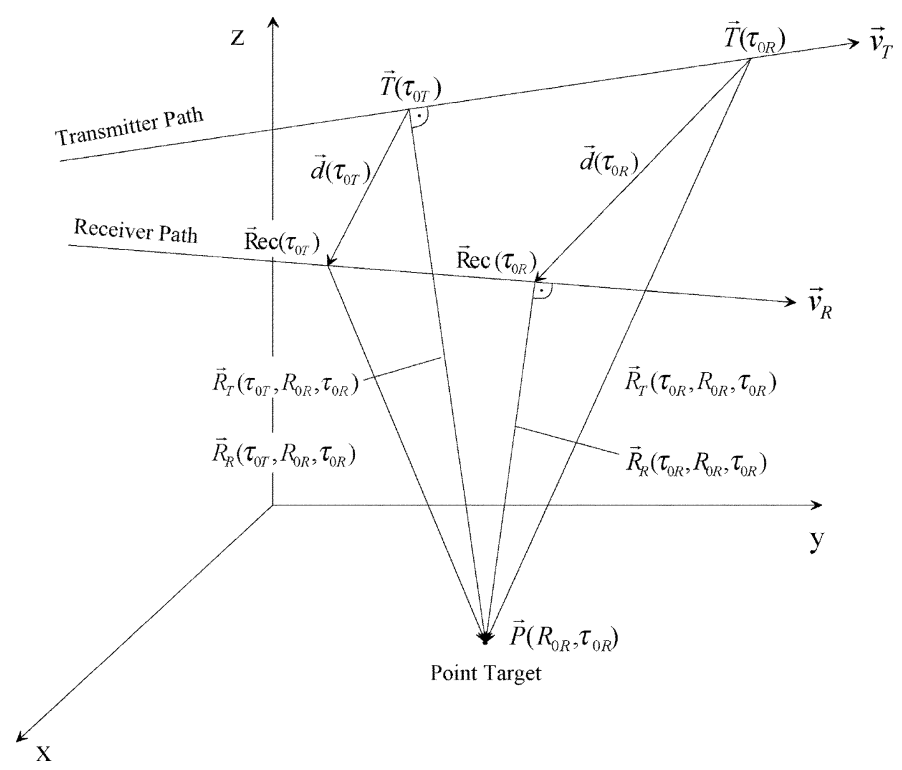

Fig. 1. Bistatic geometry.

\section{Modeling The PRoblem}

\section{A. Bistatic Geometry}

We assume a transmitter and receiver mounted on different platforms moving along different trajectories at different velocities. The geometry is shown in Fig. 1. $\tau$ denotes azimuth time, $\tau_{0}$ is the azimuth time instant when transmitter or receiver "see" the point target at closest distance [point of closest approach (PCA)], the indexes " $T$ " and " $R$ " denote transmitter and receiver values, respectively. The position $P\left(R_{0 R}, \tau_{0 R}\right)$ of the point target is implicitly specified by the time instant $\tau_{0 R}$ when it is seen perpendicularly from the receiver track (PCA) and by the corresponding slant range $R_{0 R}=\left|\vec{R}_{R}\left(\tau_{0 R}, R_{0 R}, \tau_{0 R}\right)\right|$. From the figure, we have

$$
\vec{R}_{R}\left(\tau, R_{0 R}, \tau_{0 R}\right)=\vec{R}_{R}\left(\tau_{0 R}, R_{0 R}, \tau_{0 R}\right)-\vec{v}_{R} \cdot\left(\tau-\tau_{0 R}\right)
$$

where

$$
\vec{R}_{R}\left(\tau_{0 R}, R_{0 R}, \tau_{0 R}\right) \perp \vec{v}_{R}
$$

$\vec{R}_{R}\left(\tau_{0 R}, R_{0 R}, \tau_{0 R}\right)$ is the slant range vector from receiver to point target orthogonal to the receiver velocity, which for convenience is assumed constant. $\tau_{0 R}$ is the azimuth time, when the point target is seen perpendicularly to the receiver track. Likewise, we have for the transmitter range history

$$
\vec{R}_{T}\left(\tau, R_{0 T}, \tau_{0 T}\right)=\vec{R}_{T}\left(\tau_{0 T}, R_{0 T}, \tau_{0 T}\right)-\vec{v}_{T} \cdot\left(\tau-\tau_{0 T}\right)
$$


where

$$
\vec{R}_{T}\left(\tau_{0 T}, R_{0 T}, \tau_{0 T}\right) \perp \vec{v}_{T}
$$

$\vec{R}_{T}\left(\tau_{0 T}, R_{0 T}, \tau_{0 T}\right)$ is the slant range vector from transmitter to point target orthogonal to the transmitter velocity, which for convenience is assumed constant. $\tau_{0 T}$ is the azimuth time, when the point target is seen perpendicularly to the receiver track. For the squared scalar slant range, we get from (2)

$\left|\vec{R}_{T}\left(\tau, R_{0 T}, \tau_{0 T}\right)\right|^{2}=\left|\vec{R}_{T}\left(\tau_{0 T}, R_{0 T}, \tau_{0 T}\right)\right|^{2}+v_{T}^{2} \cdot\left(\tau-\tau_{0 T}\right)^{2}$.

The transmitter motion is related to the receiver motion by

$\vec{R}_{T}\left(\tau, R_{0 R}, \tau_{0 R}\right)=\vec{R}_{R}\left(\tau_{0 R}, R_{0 R}, \tau_{0 R}\right)-\vec{v}_{T} \cdot\left(\tau-\tau_{0 R}\right)+\vec{d}\left(\tau_{0 R}\right)$

where $\vec{d}\left(\tau_{0 R}\right)=\overrightarrow{\operatorname{Re}} c\left(\tau_{0 R}\right)-\vec{T}\left(\tau_{0 R}\right)$ is the vectorial distance between transmitter and receiver at the point when the point target is seen perpendicularly to the receiver. At the azimuth point of closest receiver approach, we have from (4)

$$
\begin{gathered}
\vec{R}_{T}\left(\tau_{0 R}, R_{0 R}, \tau_{0 R}\right)=\vec{R}_{R}\left(\tau_{0 R}, R_{0 R}, \tau_{0 R}\right)+\vec{d}\left(\tau_{0 R}\right) \\
\left|\vec{R}_{T}\left(\tau_{0 R}, R_{0 R}, \tau_{0 R}\right)\right|^{2}=\left|\vec{R}_{R}\left(\tau_{0 R}, R_{0 R}, \tau_{0 R}\right)+\vec{d}\left(\tau_{0 R}\right)\right|^{2}
\end{gathered}
$$

Now, evaluating (3) at time instant $\tau_{0 R}$ and equating the result with (5), we obtain

$$
\begin{aligned}
R_{0 T}^{2} & =\left|\vec{R}_{T}\left(\tau_{0 T}, R_{0 R}, \tau_{0 R}\right)\right|^{2} \\
& =\left|\vec{R}_{R}\left(\tau_{0 R}, R_{0 R}, \tau_{0 R}\right)+\vec{d}\left(\tau_{0 R}\right)\right|^{2}-v_{T}^{2} \cdot\left(\tau_{0 R}-\tau_{0 T}\right)^{2}
\end{aligned}
$$

Dividing by the squared slant range at the receiver's point of closest approach and using $\vec{e}_{R}\left(\tau_{0 R}, R_{0 R}, \tau_{0 R}\right)=$ $\vec{R}_{R}\left(\tau_{0 R}, R_{0 R}, \tau_{0 R}\right) / R_{0 R}$, we obtain the relation

$$
\begin{aligned}
\frac{R_{0 T}^{2}}{R_{0 R}^{2}} & =\left|\frac{\vec{R}_{T}\left(\tau_{0 T}, R_{0 R}, \tau_{0 R}\right)}{\vec{R}_{R}\left(\tau_{0 R}, R_{0 R}, \tau_{0 R}\right)}\right|^{2} \\
& =\left|\vec{e}_{R}\left(\tau_{0 R}, R_{0 R}, \tau_{0 R}\right)+\frac{\vec{d}\left(\tau_{0 R}\right)}{R_{0 R}}\right|^{2}-v_{T}^{2} \cdot \frac{\left(\tau_{0 R}-\tau_{0 T}\right)^{2}}{R_{0 R}^{2}}
\end{aligned}
$$

which will be important for understanding some parameters later [cf. (29)].

\section{B. Slant Range Histories}

For the complete scalar range history, we have then

$$
R_{g}\left(\tau, R_{0 R}, \tau_{0 R}\right)=R_{R}\left(\tau, R_{0 R}, \tau_{0 R}\right)+R_{T}\left(\tau, R_{0 R}, \tau_{0 R}\right)
$$

indicating that transmitter (illuminator) and receiver individually contribute to the overall slant range histories.

Fig. 2 shows the outcoming overall slant range histories over azimuth time for a point target at different $R_{0 R}$ values. The parameters are given in Section IV. The minimum range was subtracted in each curve. The dashed curve shows the receiver slant range history multiplied by two. We note that the receiver's azimuth point of closest approach is at $1 \mathrm{~s}$.

Inspecting Fig. 2, we note the following three observations.

1) The overall slant range histories lose their hyperbolic form and look different from the receiver slant range history.

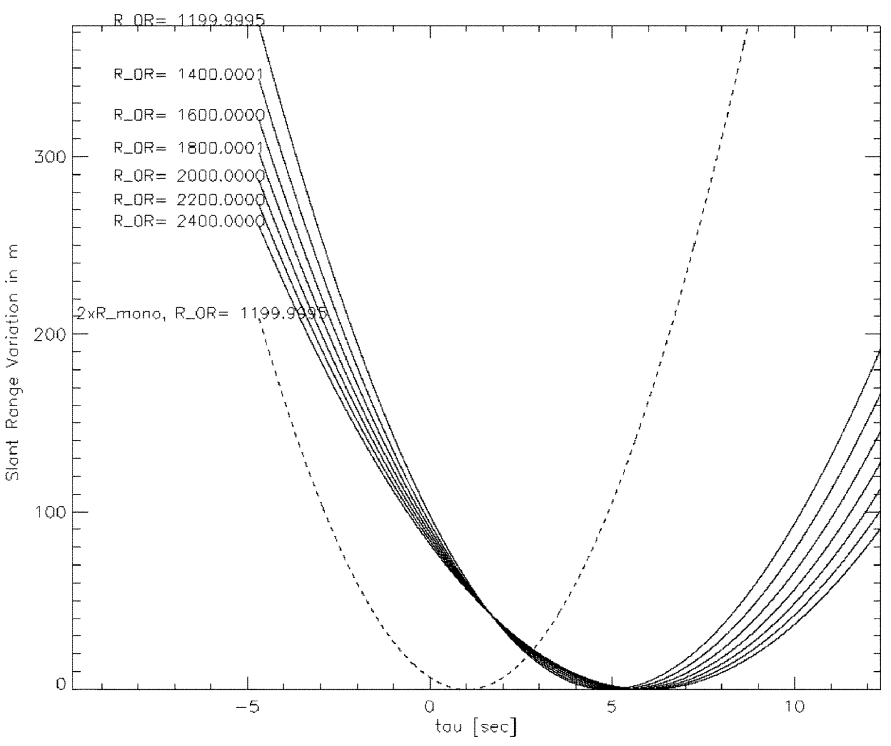

Fig. 2. Bistatic slant range histories for different point target slant ranges.

The reason for this is that the sum of two hyperbolas is no longer a hyperbola. Usually, the shape is flattened; we speak of a flat-top hyperbola.

2) The azimuth points, where the overall slant range is minimum, vary with $R_{0 R}$; we observe an azimuth shift of the minimum.

3) The shape of the overall slant range history changes in quality and not only in scaling.

As a drawback of these observations, the bistatic overall slant range history is essentially different from the monostatic slant range history; hence, bistatic SAR processing cannot be achieved by purely monostatic approaches.

\section{Range Rate Histories}

From (8), we see that the Doppler history (being proportional to the range rate history) of a point target actually consists of the two individual contributions of transmitter and receiver

$$
\dot{R}_{g}\left(\tau, R_{0 R}, \tau_{0 R}\right)=\dot{R}_{R}\left(\tau, R_{0 R}, \tau_{0 R}\right)+\dot{R}_{T}\left(\tau, R_{0 R}, \tau_{0 R}\right)
$$

For the individual scalar range rates, we have then

$$
\begin{aligned}
\dot{R}_{R}\left(\tau, R_{0 R}, \tau_{0 R}\right) \cdot R_{R}\left(\tau, R_{0 R}, \tau_{0 R}\right)= & \dot{\vec{R}}_{R}\left(\tau, R_{0 R}, \tau_{0 R}\right) \\
& \cdot \vec{R}_{R}\left(\tau, R_{0 R}, \tau_{0 R}\right) \\
= & -\vec{v}_{R} \cdot \vec{R}_{R}\left(\tau, R_{0 R}, \tau_{0 R}\right)
\end{aligned}
$$

$$
\begin{aligned}
\dot{R}_{T}\left(\tau, R_{0 R}, \tau_{0 R}\right) \cdot R_{T}\left(\tau, R_{0 R}, \tau_{0 R}\right)= & \dot{\vec{R}}_{T}\left(\tau, R_{0 R}, \tau_{0 R}\right) \\
& \cdot \vec{R}_{T}\left(\tau, R_{0 R}, \tau_{0 R}\right) \\
= & -\vec{v}_{T} \cdot \vec{R}_{T}\left(\tau, R_{0 R}, \tau_{0 R}\right)
\end{aligned}
$$

If the transmitter path were used as a reference, analogue to (10) we would have

$\underbrace{\dot{R}_{T}\left(\tau, R_{0 T}, \tau_{0 T}\right) \cdot R_{T}\left(\tau, R_{0 T}, \tau_{0 T}\right)}_{v_{T}^{2} \cdot\left(\tau-\tau_{0 T}\right)}=-\vec{v}_{T} \cdot \vec{R}_{T}\left(\tau, R_{0 T}, \tau_{0 T}\right)$. 
At time $\tau_{0 R}$ this gives

$$
v_{T}^{2} \cdot\left(\tau_{0 R}-\tau_{0 T}\right)=-\vec{v}_{T} \cdot\left(\vec{R}_{R}\left(\tau_{0 R}, R_{0 R}, \tau_{0 R}\right)+\vec{d}\left(\tau_{0 R}\right)\right)
$$

from which we get the time difference between the individual points of closest approach [cf. (29)]

$$
\tau_{0 R}-\tau_{0 T}=-\frac{\vec{v}_{T} \cdot\left(\vec{R}_{R}\left(\tau_{0 R}, R_{0 R}, \tau_{0 R}\right)+\vec{d}\left(\tau_{0 R}\right)\right)}{v_{T}^{2}}=-a_{0} .
$$

\section{Point TARget Response}

Assuming that the receiver and transmitter are perfectly synchronized, ${ }^{1}$ the signal delay from transmitter to point target and back to the receiver is given by

$$
t_{0}\left(\tau, R_{0 R}, \tau_{0 R}\right)=\frac{R_{T}\left(\tau, R_{0 R}, \tau_{0 R}\right)+R_{R}\left(\tau, R_{0 R}, \tau_{0 R}\right)}{c}
$$

and the received signal from a point target at $\left(R_{0 R}, \tau_{0 R}\right)$ after down conversion is given by

$$
\begin{aligned}
& g_{l}\left(t, \tau, R_{0 R}, \tau_{0 R}\right)=\sigma\left(R_{0 R}, \tau_{0 R}\right) \cdot w\left(\tau-\tau_{c b}\right) \\
& \quad s_{l}\left(t-t_{0}\left(\tau, R_{0 R}, \tau_{0 R}\right)\right) \cdot \exp \left[-j 2 \pi f_{0} t_{0}\left(\tau, R_{0 R}, \tau_{0 R}\right)\right] .
\end{aligned}
$$

$t$ is the range time, $w\left(\tau-\tau_{c b}\right)$ is the window centered on $\tau_{c b}$ on the azimuth time axis describing the time interval in which the point target is both illuminated by the transmitter and within the antenna footprint of the receiver. After Fourier transforming from range time domain to range frequency domain, we obtain

$$
\begin{aligned}
G_{l}\left(f, \tau, R_{0 R}, \tau_{0 R}\right)= & \sigma\left(R_{0 R}, \tau_{0 R}\right) \cdot w\left(\tau-\tau_{c b}\right) \cdot S_{l}(f) \\
& \cdot \exp \left[-j 2 \pi \frac{\left(f+f_{0}\right)}{c} \cdot R_{T}\left(\tau, R_{0 R}, \tau_{0 R}\right)\right] \\
& \cdot \exp \left[-j 2 \pi \frac{\left(f+f_{0}\right)}{c} \cdot R_{R}\left(\tau, R_{0 R}, \tau_{0 R}\right)\right] .
\end{aligned}
$$

Again we see the individual contributions of transmitter and receiver in (17). Transforming from azimuth time to azimuth frequency domain, we get

$$
G_{l}\left(f, f_{\tau}, R_{0 R}, \tau_{0 R}\right)=\sigma\left(R_{0 R}, \tau_{0 R}\right) \cdot S_{l}(f) \cdot I\left(f, f_{\tau}, R_{0 R}, \tau_{0 R}\right)
$$

where

$$
\begin{aligned}
I\left(f, f_{\tau}, R_{0 R}, \tau_{0 R}\right)= & \int_{-\infty}^{\infty} w\left(\tau-\tau_{c b}\right) \\
& \cdot \exp \left[-j \phi_{b}\left(\tau, f_{\tau}, R_{0 R}, \tau_{0 R}\right)\right] d \tau \quad(19) \\
\phi_{b}\left(\tau, f_{\tau}, R_{0 R}, \tau_{0 R}\right)= & \phi_{T}\left(\tau, f_{\tau}, R_{0 R}, \tau_{0 R}\right)+\phi_{R}\left(\tau, f_{\tau}, R_{0 R}, \tau_{0 R}\right) \\
= & 2 \pi \cdot\left[\frac{\left(f+f_{0}\right)}{c} \cdot R_{T}\left(\tau, R_{0 R}, \tau_{0 R}\right)+\frac{f_{\tau} \cdot \tau}{2}\right] \\
& +2 \pi \cdot\left[\frac{\left(f+f_{0}\right)}{c} \cdot R_{R}\left(\tau, R_{0 R}, \tau_{0 R}\right)+\frac{f_{\tau} \cdot \tau}{2}\right] .
\end{aligned}
$$

\footnotetext{
${ }^{1}$ The synchronization of transmitter and receiver over large and time-varying distances is not a trivial task.
}

Substituting (20) into (19) we obtain

$$
\begin{aligned}
I\left(f, f_{\tau}, R_{0 R}, \tau_{0 R}\right)= & \int_{-\infty}^{\infty} w\left(\tau-\tau_{c b}\right) \\
& \cdot \exp \left[-j\left(\phi_{T}\left(\tau, f_{\tau}\right)+\phi_{R}\left(\tau, f_{\tau}\right)\right)\right] d \tau \\
\cong & \exp \left[-j\left(\phi_{T}\left(\tilde{\tau}_{T}, f_{\tau}\right)+\phi_{R}\left(\tilde{\tau}_{R}, f_{\tau}\right)\right)\right] \\
& \cdot I_{2}\left(f, f_{\tau}\right)
\end{aligned}
$$

where

$$
\begin{aligned}
I_{2}\left(f, f_{\tau}\right)= & \int_{-\infty}^{\infty} w\left(\tau-\tau_{c b}\right) \\
& \cdot \exp \left[-\frac{j}{2} \cdot\left(\begin{array}{l}
\ddot{\phi}_{T}\left(\tilde{\tau}_{T}\right) \cdot\left(\tau-\tilde{\tau}_{T}\right)^{2} \\
\ddot{\phi}_{R}\left(\tilde{\tau}_{R}\right) \cdot\left(\tau-\tilde{\tau}_{R}\right)^{2}
\end{array}\right)\right] d \tau .
\end{aligned}
$$

$\tilde{\tau}_{R}, \tilde{\tau}_{T}$ are the points of stationary phases of receiver and transmitter phase histories given by

$$
\begin{aligned}
& \tilde{\tau}_{T}: \dot{\phi}_{T}\left(\tilde{\tau}_{T}, f_{\tau}, R_{0 R}, \tau_{0 R}\right)=0 \\
& \tilde{\tau}_{R}: \dot{\phi}_{R}\left(\tilde{\tau}_{R}, f_{\tau}, R_{0 R}, \tau_{0 R}\right)=0 .
\end{aligned}
$$

The quadratic Taylor series expansion around the point of stationary phase is an implicit assumption when employing the method of stationary phase to calculate a monostatic point target reference spectrum. Here, we have two halves of two monostatic phase histories that are expanded in two Taylor series around the individual points of stationary phase.

In order to find the constraints for the validity of that expansion, the range rate contributions of the individual terms are considered (Appendix). We require that the second-order range rate contributions must be negligible against the linear terms in the common point of stationary phase and that the fourth-order Lagrangian error term (summing up all the higher order terms) of the Taylor series expansion must be negligible against the third-order term. A very conservative formulation of the constraints, derived in the Appendix, is

$$
\begin{aligned}
\left|\tilde{\tau}-\tilde{\tau}_{T}\right|^{2} \stackrel{!}{\ll} 9 \cdot\left|\tilde{\tau}_{T}-\tau_{0 T}\right|^{2} \stackrel{!}{\ll} \frac{18}{7} \cdot \frac{R_{0 T}^{2}}{v_{T}^{2}} \\
\left|\tilde{\tau}-\tilde{\tau}_{r}\right|^{2} \stackrel{!}{\ll} 9 \cdot\left|\tilde{\tau}_{R}-\tau_{0 R}\right|^{2} \stackrel{!}{\ll} \frac{18}{7} \cdot \frac{R_{0 R}^{2}}{v_{R}^{2}} .
\end{aligned}
$$

Now, reasoning that the sum of two quadratic functions is again a (shifted and scaled) quadratic function, we may write for the exponent in (22) as in (25), shown at the bottom of the next page, where the common (bistatic) point of stationary phase is given by

$$
\tilde{\tau}=\frac{\ddot{\phi}_{T}\left(\tilde{\tau}_{T}\right) \cdot \tilde{\tau}_{T}+\ddot{\phi}_{R}\left(\tilde{\tau}_{R}\right) \cdot \tilde{\tau}_{R}}{\ddot{\phi}_{T}\left(\tilde{\tau}_{T}\right)+\ddot{\phi}_{R}\left(\tilde{\tau}_{R}\right)} .
$$

Solving (22) by the method of stationary phase and substituting (25) and (26), we obtain from (19)

$I\left(f, f_{\tau}, R_{0 R}, \tau_{0 R}\right) \cong w\left(\tilde{\tau}-\tau_{c b}\right)$

$$
\cdot \exp [-j \underbrace{\left(\phi_{T}\left(\tilde{\tau}_{T}, f_{\tau}\right)+\phi_{R}\left(\tilde{\tau}_{R}, f_{\tau}\right)\right)}_{\Psi_{1}\left(f, f_{\tau}\right)}]
$$




$$
\begin{gathered}
\cdot \exp [-\frac{j}{2} \underbrace{\frac{\ddot{\phi}_{T}\left(\tilde{\tau}_{T}\right) \cdot \ddot{\phi}_{R}\left(\tilde{\tau}_{R}\right)}{\ddot{\phi}_{T}\left(\tilde{\tau}_{T}\right)+\ddot{\phi}_{R}\left(\tilde{\tau}_{R}\right)} \cdot\left(\tilde{\tau}_{T}-\tilde{\tau}_{R}\right)^{2}}_{\Psi_{2}\left(f, f_{\tau}\right)} \\
\cdot \frac{\sqrt{2 \pi}}{\sqrt{\ddot{\phi}_{T}\left(\tilde{\tau}_{T}\right)+\ddot{\phi}_{R}\left(\tilde{\tau}_{R}\right)}} \cdot \exp \left(-j \frac{\pi}{4}\right) \cdot(27)
\end{gathered}
$$

Equation (27) conceptually shows how we can combine the two monostatic phase and Doppler histories of transmitter and receiver to the bistatic point target reference spectrum. We have to calculate the individual points of stationary phase, the corresponding phase arguments in those points, and the second phase derivatives. For the receiver, the calculation is straightforward and has been documented in the open literature. When considering the transmitter, the coupling expressed in (4) is essential. Yet the calculation and combination of the individual terms is lengthy and must be omitted. The stationary points clearly depend on the azimuth frequency; hence, the first term in (27)-w( $\left.\tilde{\tau}-\tau_{c b}\right)$-defines azimuth bandwidth and bistatic Doppler centroid frequency as the center frequency of the azimuth reference spectrum. The analysis of this window is lengthy, and exact equations determining Doppler bandwidth and Doppler Centroid are hard to find. Approximate solutions are given in [8]. The first exponential term is obtained as

$$
\begin{aligned}
\Psi_{1}\left(f, f_{\tau}\right)= & \phi_{T}\left(\tilde{\tau}_{T}, f_{\tau}\right)+\phi_{R}\left(\tilde{\tau}_{R}, f_{\tau}\right)=\pi f_{\tau} \cdot\left(2 \tau_{0 R}+a_{0}\right) \\
& +2 \pi \frac{R_{0 R}}{c} \cdot\left[\sqrt{\underbrace{\left(f+f_{0}\right)^{2}-f_{\tau}^{2} \frac{c^{2}}{4 v_{R}^{2}}}_{F_{R}\left(f, f_{\tau}\right)}}\right. \\
& \left.+\sqrt{\underbrace{\left(f+f_{0}\right)^{2}-f_{\tau}^{2} \frac{c^{2}}{4 v_{T}^{2}}}_{F_{T}\left(f, f_{\tau}\right)}} \cdot a_{2}\right]
\end{aligned}
$$

where the coefficients $a_{0}, a_{2}$ determining the "bistatic grade" are given by [cf. (7) and (14)]

$$
\begin{aligned}
a_{0} & =\frac{\left(\vec{R}_{R}\left(\tau_{0 R}, R_{0 R}, \tau_{0 R}\right)+\vec{d}\left(\tau_{0 R}\right)\right) \cdot \vec{v}_{T}}{v_{T}^{2}}=\tau_{0 T}-\tau_{0 R} \\
a_{2} & =\sqrt{\left(\vec{e}_{R}\left(\tau_{0 R}, R_{0 R}, \tau_{0 R}\right)+\frac{\vec{d}\left(\tau_{0 R}\right)}{R_{0 R}}\right)^{2}-v_{T}^{2} \cdot \frac{a_{0}^{2}}{R_{0 R}^{2}}} \\
& =\frac{R_{0 T}}{R_{0 R}} .
\end{aligned}
$$

In the monostatic case, we get

$$
\begin{aligned}
\lim _{d->0, \vec{v}_{T} \perp \vec{R}_{R}\left(\tau_{0 R}, R_{0 R}, \tau_{0 R}\right)} & a_{0}=0 \\
\lim _{d->0, \vec{v}_{T} \perp \vec{R}_{R}\left(\tau_{0 R}, R_{0 R}, \tau_{0 R}\right)} & a_{2}=1
\end{aligned}
$$

and the first exponential term (28) becomes the familiar monostatic point target reference function. After some lengthy algebra, the second exponential phase term is found

$$
\begin{aligned}
\Psi_{2}\left(f, f_{\tau}\right)= & \frac{\ddot{\phi}_{T}\left(\tilde{\tau}_{T}\right) \cdot \ddot{\phi}_{R}\left(\tilde{\tau}_{R}\right)}{\ddot{\phi}_{T}\left(\tilde{\tau}_{T}\right)+\ddot{\phi}_{R}\left(\tilde{\tau}_{R}\right)} \cdot\left(\tilde{\tau}_{T}-\tilde{\tau}_{R}\right)^{2} \\
= & \frac{2 \pi}{R_{0 R} c} \cdot \frac{v_{R}^{2} v_{T}^{2}}{\left(f+f_{0}\right)^{2}} \\
& \cdot \frac{F_{R}\left(f, f_{\tau}\right)^{3 / 2} \cdot F_{T}\left(f, f_{\tau}\right)^{3 / 2}}{v_{T}^{2} \cdot F_{T}\left(f, f_{\tau}\right)^{3 / 2}+a_{2} \cdot v_{R}^{2} \cdot F_{R}\left(f, f_{\tau}\right)^{3 / 2}} \\
& \cdot\left(\tilde{\tau}_{T}-\tilde{\tau}_{R}\right)^{2}
\end{aligned}
$$

where the difference between the stationary points is

$$
\begin{aligned}
\left(\tilde{\tau}_{T}-\tilde{\tau}_{R}\right)^{2}= & f_{\tau}^{2} \cdot\left(\frac{c}{2 v_{R}^{2} v_{T}^{2}}\right)^{2} \cdot \frac{R_{0 R}^{2}}{F_{T}\left(f, f_{\tau}\right) \cdot F_{R}\left(f, f_{\tau}\right)} \\
& \cdot\left[v_{R}^{2} \cdot F_{R}^{1 / 2}\left(f, f_{\tau}\right) \cdot a_{2}-v_{T}^{2} \cdot F_{T}^{1 / 2}\left(f, f_{\tau}\right)\right]^{2} \\
& +a_{0}^{2}-2 a_{0} f_{\tau} \cdot \frac{c}{2 v_{R}^{2} v_{T}^{2}} \cdot \frac{R_{0 R}}{\sqrt{F_{T}\left(f, f_{\tau}\right) \cdot F_{R}\left(f, f_{\tau}\right)}} \\
& \cdot\left[v_{R}^{2} \cdot F_{R}^{1 / 2}\left(f, f_{\tau}\right) \cdot a_{2}-v_{T}^{2} \cdot F_{T}^{1 / 2}\left(f, f_{\tau}\right)\right] \\
= & {\left[a_{0}-f_{\tau} \cdot \frac{c}{2 v_{R}^{2} v_{T}^{2}} \cdot \frac{R_{0 R}}{\sqrt{F_{T}\left(f, f_{\tau}\right) \cdot F_{R}\left(f, f_{\tau}\right)}}\right.} \\
& \left.\cdot\left[v_{R}^{2} \cdot F_{R}^{1 / 2}\left(f, f_{\tau}\right) \cdot a_{2}-v_{T}^{2} \cdot F_{T}^{1 / 2}\left(f, f_{\tau}\right)\right]\right]^{2}
\end{aligned}
$$

In the monostatic case, the right-hand side of (32) vanishes, the stationary points of transmitter and receiver coincide, and the second phase term vanishes identically. Summarizing, we note that the bistatic reference spectrum given in (27) "collapses" to the monostatic reference spectrum if the bistatic baseline goes to zero and if the velocities are equal.

\section{RESULTS}

For the simulation, we used the parameters given in Table I. The parameters were chosen different from [4] to consider an airborne case with a high bistatic degree expressed by a bistatic angle of $25^{\circ}$.

We first generated the reference spectrum of a point target at slant range $R_{0 R}=1800 \mathrm{~m}$. The receiver's off-nadir angle was $56.25^{\circ}$. The receiver track was parallel to the $y$ axis, and

$$
\ddot{\phi}_{T}\left(\tilde{\tau}_{T}\right) \cdot\left(\tau-\tilde{\tau}_{T}\right)^{2}+\ddot{\phi}_{R}\left(\tilde{\tau}_{R}\right) \cdot\left(\tau-\tilde{\tau}_{R}\right)^{2}=\frac{\ddot{\phi}_{T}\left(\tilde{\tau}_{T}\right) \cdot \ddot{\phi}_{R}\left(\tilde{\tau}_{R}\right)}{\ddot{\phi}_{T}\left(\tilde{\tau}_{T}\right)+\ddot{\phi}_{R}\left(\tilde{\tau}_{R}\right)} \cdot\left(\tilde{\tau}_{T}-\tilde{\tau}_{R}\right)^{2}+\left(\ddot{\phi}_{T}\left(\tilde{\tau}_{T}\right)+\ddot{\phi}_{R}\left(\tilde{\tau}_{R}\right)\right) \cdot(\tau-\tilde{\tau})^{2}
$$


TABLE I

SIMULATION PARAMETERS

\begin{tabular}{|c|c|c|}
\hline Parameter & Symbol & Value \\
\hline Center Frequency & $\mathrm{f}_{0}$ & $10 \mathrm{GHz}$ \\
\hline Receiver Height & $\mathrm{h}_{\mathrm{R}}$ & $1000 \mathrm{~m}$ \\
\hline Transmitter Height & $\mathrm{h}_{\mathrm{T}}$ & $1035 \mathrm{~m}$ \\
\hline Receiver Velocity & $v_{R}$ & $90 \mathrm{~ms}^{-1}$ \\
\hline Transmitter Velocity & $\mathrm{v}_{\mathrm{T}}$ & $80 \mathrm{~ms}^{-1}$ \\
\hline Slant Range Resolution & $\Delta_{R}$ & $0.1 \mathrm{~m}$ \\
\hline Azimuth Resolution (nom) & $\Delta_{a}$ & $0.1 \mathrm{~m}$ \\
\hline $\begin{array}{l}\text { Azimuth Time of Receiver's Point } \\
\text { of Closest Approach }\end{array}$ & $\tau_{0 R}$ & $1 \mathrm{~s}$ \\
\hline $\begin{array}{l}\text { Azimuth Center Time of Common } \\
\text { Window }\end{array}$ & $\tau_{c b}$ & $1 \mathrm{~s}$ \\
\hline $\begin{array}{l}\text { Receiver Slant Range at Point of } \\
\text { Closest Approach }\end{array}$ & $\mathrm{R}_{0 \mathrm{R}}$ & $1800 \mathrm{~m}$ \\
\hline $\begin{array}{l}\text { Angle between Transmitter and } \\
\text { Receiver Velocity Vector }\end{array}$ & & $1^{\circ}$ \\
\hline Time Difference Parameter & $a_{0}$ & $14.82 \mathrm{~s}$ \\
\hline Slant Range Ratio Parameter & $a_{2}$ & 2.125 \\
\hline Doppler Centroid & $f_{D C}$ & $808 \mathrm{~Hz}$ \\
\hline $\begin{array}{l}\text { Baseline Vector at Receiver's } \\
\text { Azimuth Point of Closest Approach }\end{array}$ & $\vec{d}\left(\tau_{0 R}\right)$ & {$\left[\begin{array}{c}2164,85 \\
1248,88 \\
35\end{array}\right] \mathrm{m}$} \\
\hline $\begin{array}{l}\text { Bistatic Angle at Receiver's Point } \\
\text { of closest Approach }\end{array}$ & $\beta\left(\tau_{0 R}\right)$ & $25.34^{\circ}$ \\
\hline
\end{tabular}

the angle between transmitter track and $y$ axis was $1^{\circ}$ (counterclockwise). The baseline component of $1.248 \mathrm{~km}$ in the $y$ direction introduces a considerable Doppler centroid. Fig. 3 shows the corresponding bistatic point target response. We see two parts of a chirp separated spatially and corresponding to the individual contributions of transmitter and receiver.

Inspecting Fig. 3 it becomes obvious that the bistatic point target response looks quite different from the monostatic one. The main reason for this is the range history which-depending on the bistatic "degree"-is quite different from the monostatic case. Fig. 4 shows the convolution partners that form the bistatic range migration (flat-top hyperbola), the elliptic term corresponding to the phasor in (34), and the quasi-monostatic hyperbola corresponding to (33). Due to the large Doppler centroid, we see squinted versions of ellipse and hyperbola.

Further regarding Fig. 4, it becomes clear that the bistatic constellation is looking "ahead," and the beams are squinted forward, introducing a positive Doppler centroid (approximately $880 \mathrm{~Hz}$ ). Both convolution partners are shifted against each other in a way that the convolution of both produces a bistatic point target response that is correctly registered. Figs. 3 and 4 are in Fourier time and range representation and subject to wrap around effects, if the time signal's extent is larger than the transformation length.

\section{INTERPRETATION}

\section{A. General Case}

After back transformation into the range azimuth domain, the product of the two exponentials in (27) clearly transforms into a convolution of the corresponding range azimuth functions (cf. Fig. 4).

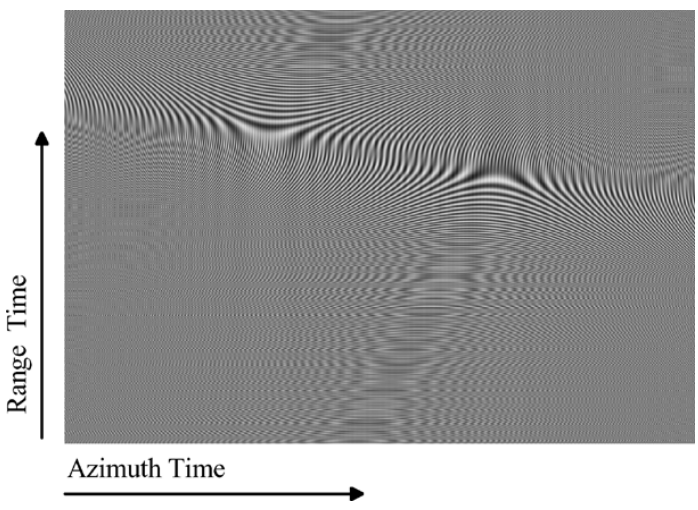

Fig. 3. Bistatic point target response (cutout).

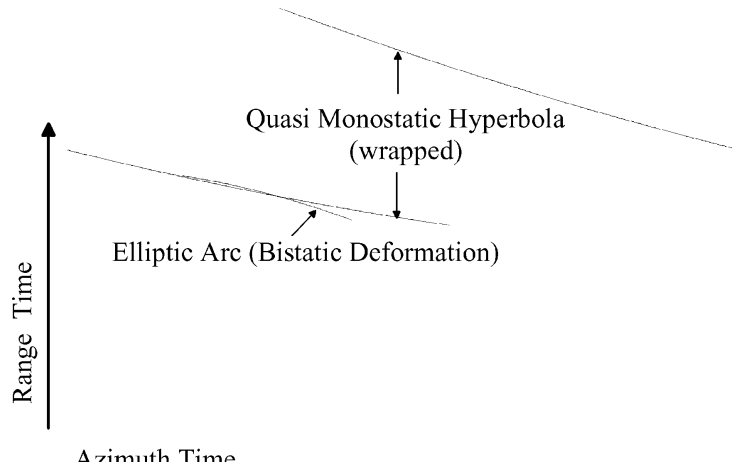

Azimuth Time

Fig. 4. Monostatic range hyperbola and bistatic correction term (elliptic arc).

1) Quasi-Monostatic Term: The first phasor term

$$
\begin{aligned}
\Psi_{1}\left(f, f_{\tau}\right)= & \phi_{T}\left(\tilde{\tau}_{T}, f_{\tau}\right)+\phi_{R}\left(\tilde{\tau}_{R}, f_{\tau}\right) \\
= & 2 \pi f_{\tau} \cdot\left(\frac{\tau_{0 R}+\tau_{0 T}}{2}\right) \\
& +2 \pi \cdot\left[\frac{R_{0 R}}{c} \sqrt{\underbrace{\left(f+f_{0}\right)^{2}-f_{\tau}^{2} \frac{c^{2}}{4 v_{R}^{2}}}_{F_{R}\left(f, f_{\tau}\right)}}\right. \\
& \left.+\frac{R_{0 T}}{c} \sqrt{\underbrace{\left(f+f_{0}\right)^{2}-f_{\tau}^{2} \frac{c^{2}}{4 v_{T}^{2}}}_{F_{T}\left(f, f_{\tau}\right)}}\right]
\end{aligned}
$$

is a quasi-monostatic expression, meaning that instead of the familiar single monostatic hyperbola, weighted by the doubled slant range $2 \cdot R_{0}$ (two-way delay), we have the sum of two semimonostatic phase histories, the first one being the one-way receiver and the second one being the one-way transmitter phase history. Due to $R_{0 R} \neq R_{0 T}$, both histories differ in amplitude, and due to $v_{R} \neq v_{T}$, they also differ in shape. The linear phase term in $f_{\tau}$ shifts the corresponding azimuth time history to the midpoint between the individual points of closest approach. $R_{0 T}$ and $\tau_{0 T}$ can be determined from (29). For nonconstant velocity vectors, both terms are range and azimuth time 
variant. The azimuth time dependence vanishes if and only if $\dot{\vec{d}}(t)=\overrightarrow{0} \Rightarrow \vec{v}_{T}=\vec{v}_{R}$

Conceptually, the sum of two half monostatic phase histories (giving rise to the product of the corresponding exponentials) transforms into the convolution of the corresponding monostatic semirange hyperbolas where the convolution partners in the general case are range and azimuth time variant.

2) Bistatic Deformation: Inspecting the range histories of a bistatic sensor shown in Fig. 2, it becomes clear that even for identical velocities, the normal bistatic range history takes on the form of a "flattened" hyperbola, a so-called flat-top hyperbola. Conceptually, this flat-top hyperbola is generated by further convolving the quasi-monostatic hyperbola with the inverse Fourier transform of the second phasor function in (27)

$$
\begin{aligned}
\Psi_{2}\left(f, f_{\tau}\right)= & \frac{2 \pi}{R_{0 R} c} \cdot \frac{v_{R}^{2} v_{T}^{2}}{\left(f+f_{0}\right)^{2}} \\
& \cdot \frac{F_{R}\left(f, f_{\tau}\right)^{3 / 2} \cdot F_{T}\left(f, f_{\tau}\right)^{3 / 2}}{v_{T}^{2} \cdot F_{T}\left(f, f_{\tau}\right)^{3 / 2}+a_{2} \cdot v_{R}^{2} \cdot F_{R}\left(f, f_{\tau}\right)^{3 / 2}} \\
& \cdot\left(\tilde{\tau}_{T}-\tilde{\tau}_{R}\right)^{2}
\end{aligned}
$$

where $\left(\tilde{\tau}_{T}-\tilde{\tau}_{R}\right)^{2}$ from (32)

The inverse of the complex exponential containing (34) turns out to be an elliptic arc in the range/azimuth time domain. Conceptually, the multiplication of the two exponentials in (27) indicates that the range history of a bistatic sensor can be expressed as a convolution of a monostatic SAR sensor's range history with a small part of an ellipse (Fig. 4). It is important to note that the elliptic arc is complex valued, and it shows a heavily varying phase. This is to be expected. The elliptic arc and the quasi-monostatic hyperbola are Dirac lines consisting of noncountable infinities of two-dimensional Dirac impulses. During the convolution, any Dirac impulse of one line (e.g., the ellipse) reproduces a shifted replica of the other; the superposition of all shifted replicas of the hyperbola must constructively interfere along the line of the flat-top hyperbola and must vanish elsewhere. This is only possible by additional phase modulation of the elliptic arc.

Ignoring the convolution with the range chirp, we note that in the general case, a bistatic point target response can be conceptually modeled as a triple convolution. The semipoint target response of a monostatic sensor (receiver/transmitter) is first convolved with the semipoint target response of the second sensor (transmitter/receiver). The outcoming result is then further convolved with an elliptic arc (the bistatic deformation term). This elliptic arc, a generalized smile, might be considered as some generalization of "Rocca's Smile" [5], [6], which was derived for the constant offset case. Claerbout [5], [6] gives more intuitive insights into the flat-top hyperbola phenomenon; any amplitude and phase considerations are not given there.

\section{B. Identical Velocities $v_{r}=v_{T}=v_{0}$}

Letting the velocities be equal in amplitude $v_{r}=v_{T}=v_{0}$ but not in direction $\vec{v}_{R} \neq \vec{v}_{T}$ some simplifications become possible.
1) Monostatic Term: The first phasor term becomes

$$
\begin{aligned}
\Psi_{1}\left(f, f_{\tau}\right)= & \phi_{T}\left(\tilde{\tau}_{T}, f_{\tau}\right)+\phi_{R}\left(\tilde{\tau}_{R}, f_{\tau}\right) \\
= & 2 \pi f_{\tau} \cdot\left(\frac{\tau_{0 R}+\tau_{0 T}}{2}\right)+\frac{4 \pi}{c} \\
& \cdot \frac{R_{0 R}+R_{0 T}}{2} \sqrt{\left(f+f_{0}\right)^{2}-f_{\tau}^{2} \frac{c^{2}}{4 v_{0}^{2}}}
\end{aligned}
$$

being a proper monostatic expression, corresponding to a monostatically imaged point target at position

$$
\left(\frac{\tau_{0 R}+\tau_{0 T}}{2}, \frac{R_{0 R}+R_{0 T}}{2}\right)
$$

$R_{0 T}$ and $\tau_{0 T}$ are again determined from (29). $w\left(\tau-\tau_{c b}\right)$ then determines time extent and center of the cutout from the monostatic range history.

2) Bistatic Deformation: The bistatic deformation term now reduces to

$$
\Psi_{2}\left(f, f_{\tau}\right)=\frac{2 \pi}{c} \cdot \frac{v_{0}^{2}}{\left(f+f_{0}\right)^{2}} \cdot \frac{F_{T R}\left(f, f_{\tau}\right)^{3 / 2}}{R_{0 R}+R_{0 T}} \cdot\left(\tilde{\tau}_{T}-\tilde{\tau}_{R}\right)^{2}
$$

where

$$
\begin{aligned}
& \left(\tilde{\tau}_{T}-\tilde{\tau}_{R}\right)^{2}=\left[\tau_{0 T}-\tau_{0 R}-f_{\tau} \cdot \frac{c}{2 v_{0}^{2}} \cdot \frac{R_{0 T}-R_{0 R}}{\sqrt{F_{T R}\left(f, f_{\tau}\right)}}\right]^{2} \\
& F_{T R}\left(f, f_{\tau}\right)=\left(f+f_{0}\right)^{2}-f_{\tau}^{2} \frac{c^{2}}{4 v_{0}^{2}}
\end{aligned}
$$

Again the inverse Fourier transform turns out to be an elliptic arc in the range/azimuth time domain. As in the preceding case, this generalized smile changes with slant range and azimuth time as the difference between $R_{0 T}$ and $R_{0 R}$ is azimuth time variant. Since for constant velocities the difference changes linearly with the time, chirp scaling strategies to equalize the bistatic deformation term (comparable to equalizing the range migration) might be thought of.

Again ignoring the convolution with the range chirp, we note that in this case the bistatic point target response can be modeled as a double convolution. The point target response of a monostatic equivalent sensor is convolved with the generalized azimuth and range varying smile.

\section{Identical Velocity Vectors $\vec{v}_{T}=\vec{v}_{R}$ : Rocca's Smile}

1) Monostatic Term: Regarding (35), the first phasor remains unchanged, corresponding to a monostatically imaged point target at position

$$
\left(\frac{\tau_{0 R}+\tau_{0 T}}{2}, \frac{R_{0 R}+R_{0 T}}{2}\right) .
$$

Now, we have an equivalent monostatic SAR sensor being located just in the middle between transmitter and receiver and traveling on the velocity vector $\vec{v}_{0}=\vec{v}_{T}=\vec{v}_{R}$ and operating in strip mode. 
2) Bistatic Deformation: The bistatic deformation term (36) essentially remains the same except that it is now constant with respect to azimuth time but still range variant. As indicated earlier, the compensation can be accomplished blockwise in range blocks.

Again ignoring the convolution with the range chirp, we note that in this case the bistatic point target response can be modeled as a double convolution. The point target response of a monostatic equivalent sensor is convolved with Rocca's smile, which is a range varying smile. This case is treated in [7] and is considered in further detail in [8].

\section{FOCUSING}

The separation of the point target's reference function into quasi-monostatic phasor and bistatic deformation phasor readily suggests a two-step focusing approach:

Step 1) compensation of bistatic deformation;

Step 2) quasi-monostatic processing.

\section{A. General Case}

In general, the bistatic deformation term is clearly range variant and azimuth time variant; hence, it must be compensated by transforming the bistatic raw data blockwise and then multiplying with the conjugate complex term, where the slant range is adjusted to the mean slant range within the block. The quasi-monostatic processing essentially consists of two semimonostatic processing steps with the parameters adjusted to transmitter and receiver characteristics.

\section{B. Identical Velocities $v_{r}=v_{T}=v_{0}$}

If the transmitter and receiver velocity are equal in size, but not in direction, the quasi-monostatic phase history becomes a proper monostatic phase history. Yet the bistatic correction term will vary with range and azimuth. Refined scaling strategies comparable to chirp scaling or introducing scaled Fourier transformation techniques might be thought of.

\section{Identical Velocity Vectors $\vec{v}_{T}=\vec{v}_{R}$ : Rocca's Smile}

The bistatic compensation term will be constant over azimuth time, so that the block separation is only with respect to range. More refined techniques to accommodate the range variance might be thought of, such as nonlinear interpolations, scaling procedures. In this case, the monostatic processing step is quite conventional and can be realized by any monostatic processing algorithm.

\section{APPENDIX}

The range rate errors (corresponding to the instantaneous frequency errors) of the Taylor series expansions of transmitter (index $T$ ) and receiver (index $R$ ) are given by

$$
\begin{aligned}
e_{T, R}(\tau)=\frac{1}{2 !} \dddot{R}_{T, R}\left(\tilde{\tau}_{T, R}\right) \cdot\left(\tau-\tilde{\tau}_{T, R}\right)^{2} & \\
& +\frac{1}{3 !} \cdot R_{T, R}^{(4)}\left(\xi_{T, R}\right) \cdot\left(\tau-\tilde{\tau}_{T, R}\right)^{3}
\end{aligned}
$$

where the dependencies on $R_{0 T}$ and $R_{0 R}, \tau_{0 T}$ and $\tau_{0 R}$ have been omitted for convenience. $\tilde{\tau}_{T, R}$ are the individual points of stationary phase of transmitter $(T)$ and receiver $(R) . \xi_{T, R}$ are two different points within the intervals $\left(\tilde{\tau}_{T, R}, \tau\right)$ summarizing the integrated error terms (of transmitter and receiver) according to the mean value theorem. Now, we require that the range rate error in the common point of stationary phase is negligible against the nominal value

$$
\left|\frac{\frac{1}{2 !} \dddot{R}_{T, R}\left(\tilde{\tau}_{T, R}\right) \cdot\left(\tilde{\tau}-\tilde{\tau}_{T, R}\right)+\frac{1}{3 !} \cdot R_{T, R}^{(4)}\left(\xi_{T, R}\right) \cdot\left(\tilde{\tau}-\tilde{\tau}_{T, R}\right)^{2}}{\ddot{R}_{T, R}\left(\tilde{\tau}_{T, R}\right)}\right| \stackrel{!}{\ll} 1
$$

which can be guaranteed if

$$
\frac{\dddot{R}_{T, R}\left(\tilde{\tau}_{T, R}\right) \cdot\left(\tilde{\tau}-\tilde{\tau}_{T, R}\right)}{\ddot{R}_{T, R}\left(\tilde{\tau}_{T, R}\right)} \stackrel{!}{\ll} 2
$$

and if the quadratic term in (39) is small against the linear term

$$
\left|\frac{R_{T, R}^{(4)}\left(\xi_{T, R}\right) \cdot\left(\tilde{\tau}-\tilde{\tau}_{T, R}\right)}{\dddot{R}_{T, R}\left(\tilde{\tau}_{T, R}\right)}\right| \stackrel{!}{\ll} 3 .
$$

Starting with (41) and substituting the third- and fourth-order derivatives of the transmitter and receiver range histories [cf. (3)], we get the constraint

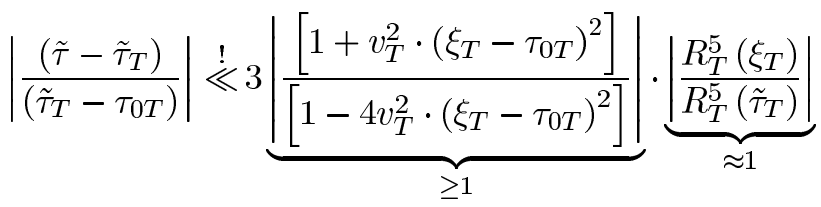

$$
\begin{aligned}
& \Rightarrow \underbrace{\left|\frac{\left(\tilde{\tau}-\tilde{\tau}_{T}\right)}{\left(\tilde{\tau}_{T}-\tau_{0 T}\right)}\right| \stackrel{!}{\ll} 3}_{\text {Transmitter }} \text { and } \underbrace{\left|\frac{\left(\tilde{\tau}-\tilde{\tau}_{R}\right)}{\left(\tilde{\tau}_{R}-\tau_{0 R}\right)}\right| \stackrel{!}{\ll} 3}_{\text {Receiver }}
\end{aligned}
$$

where the constraint for the receiver was derived in an analogue way. Equation (42) states that the distance between the common point of stationary phase and the individual points of stationary phase should be much smaller than three times the distance between the individual points of stationary phase and the corresponding points of closest approach. Now, using (40) and substituting the second- and third-order derivatives of the slant range histories, we get

$$
\begin{aligned}
& \left|\tilde{\tau}_{T}-\tilde{\tau}_{0 T}\right| \cdot\left|\tilde{\tau}-\tilde{\tau}_{T}\right| \stackrel{!}{\ll} \frac{2}{3} \cdot \frac{R_{0 T}^{2}+v_{T}^{2}\left(\tilde{\tau}_{T}-\tau_{0 T}\right)^{2}}{v_{T}^{2}} \\
& \left|\tilde{\tau}_{T}-\tilde{\tau}_{0 R}\right| \cdot\left|\tilde{\tau}-\tilde{\tau}_{R}\right| \stackrel{!}{\ll} \frac{2}{3} \cdot \frac{R_{0 R}^{2}+v_{R}^{2}\left(\tilde{\tau}_{R}-\tau_{0 R}\right)^{2}}{v_{R}^{2}} .
\end{aligned}
$$

Now, (42) and (43) form a system of inequalities that can be solved graphically, identifying the regions of solution for a given constellation.

Here, we proceed in a more conservative way, seeking for a solution which generally holds. From (42), we conclude that

$$
\begin{gathered}
\left|\left(\tilde{\tau}-\tilde{\tau}_{T}\right)\right| \cdot\left|\tilde{\tau}_{T}-\tau_{0 T}\right| \stackrel{!}{\ll} 3 \cdot\left(\tilde{\tau}_{T}-\tau_{0 T}\right)^{2} \\
\left|\left(\tilde{\tau}-\tilde{\tau}_{R}\right)\right| \cdot\left|\tilde{\tau}_{R}-\tau_{0 R}\right| \stackrel{!}{\ll} 3 \cdot\left(\tilde{\tau}_{R}-\tau_{0 R}\right)^{2}
\end{gathered}
$$


can be used as upper limits for the left-hand sides in (43), from which we then obtain the sharpened requirement

$$
\begin{aligned}
\left|\tilde{\tau}_{T}-\tau_{0 T}\right|^{2} & \stackrel{!}{\ll} \frac{2}{9} \cdot \frac{R_{0 T}^{2}+v_{T}^{2}\left(\tilde{\tau}_{T}-\tau_{0 T}\right)^{2}}{v_{T}^{2}} \\
& \Rightarrow\left|\tilde{\tau}_{T}-\tau_{0 T}\right|^{2} \stackrel{!}{\ll} \frac{2}{7} \cdot \frac{R_{0 T}^{2}}{v_{T}^{2}} \\
\left|\tilde{\tau}_{R}-\tau_{0 R}\right|^{2} & \stackrel{!}{\ll} \frac{2}{9} \cdot \frac{R_{0 R}^{2}+v_{R}^{2}\left(\tilde{\tau}_{R}-\tau_{0 R}\right)^{2}}{v_{R}^{2}} \\
& \Rightarrow\left|\tilde{\tau}_{R}-\tau_{0 R}\right|^{2} \stackrel{!}{\ll} \frac{2}{7} \cdot \frac{R_{0 R}^{2}}{v_{R}^{2}} .
\end{aligned}
$$

Resubstituting (42), we obtain

$$
\begin{aligned}
\left|\tilde{\tau}-\tilde{\tau}_{T}\right|^{2} \stackrel{!}{\ll} 9 \cdot\left|\tilde{\tau}_{T}-\tau_{0 T}\right|^{2} \stackrel{!}{\ll} \frac{18}{7} \cdot \frac{R_{0 T}^{2}}{v_{T}^{2}} \\
\left|\tilde{\tau}-\tilde{\tau}_{r}\right|^{2} \stackrel{!}{\ll} 9 \cdot\left|\tilde{\tau}_{R}-\tau_{0 R}\right|^{2} \stackrel{!}{\ll} \frac{18}{7} \cdot \frac{R_{0 R}^{2}}{v_{R}^{2}}
\end{aligned}
$$

as constraints that guarantee the validity of the double Taylor series expansion. From (46), we get the following:

$$
\begin{aligned}
& \text { Airborne Case: } R_{0 T}=5 \mathrm{~km} ; v_{T}=100 \mathrm{~ms}^{-1} \text { : } \\
& \left|\tilde{\tau}-\tilde{\tau}_{T}\right| \ll 80,17 \mathrm{~s} \text { and }\left|\tilde{\tau}_{T}-\tau_{0 T}\right| \ll 26,73 \mathrm{~s} ; \\
& \text { Spaceborne Case: } R_{0 T}=200 \mathrm{~km} ; v_{T}=7 \mathrm{kms}^{-1} \text { : } \\
& \left|\tilde{\tau}-\tilde{\tau}_{T}\right| \ll 45,82 \mathrm{~s} \text { and }\left|\tilde{\tau}_{T}-\tau_{0 T}\right| \ll 15,27 \mathrm{~s} .
\end{aligned}
$$

It is still possible to relax the constraints by a graphical analysis, yielding a wider range of validity.

\section{ACKNOWLEDGMENT}

When first publishing these results [4], it was F. Rocca, who called the author's attention to the flat-top hyperbola as a convolution of a hyperbola with a short elliptic arc ("Rocca's smile"), which is gratefully appreciated. The authors should furthermore like to thank the anonymous reviewers very warmly for the detailed, constructive, and encouraging criticism. The authors are especially indebted to K. Natroshvili for his support on the validity of the approach.

\section{REFERENCES}

[1] M. D'Errico and A. Moccia, "Remote sensing satellite formation for bistatic synthetic aperture radar observation," Proc. SPIE, vol. 4540, 2001.

[2] M. Soumekh, "Bistatic synthetic aperture radar imaging using widebandwidth continuous-wave sources," in Proc. SPIE, vol. 3462, 1998.

[3] N. J. Willis, Bistatic Radar. Norwood, MA: Artech House, 1991.

[4] O. Loffeld, H. Nies, V. Peters, and S. Knedlik, "Models and useful relations for bistatic SAR processing," in Proc. IGARSS, vol. III, Toulouse, France, 2003, pp. 1442-1445.

[5] J. Claerbout. Migration with velocity estimation-Rocca's smear operator. [Online]. Available: http://sepwww.stanford.edu/sep/prof/iei/vdmo/paper_html/node15.html.

[6] — - Ellipsoids versus hyperboloids. [Online]. Available: http:// sepwww.stanford.edu/public/docs/sep89/jon2/paper_html/index.html.

[7] D. D'Aria, A. M. Guarnieri, and F. Rocca, "Focusing bistatic synthetic aperture radar using dip move out," IEEE Trans. Geosci. Remote Sensing, vol. 42, pp. 1362-1376, July 2004.

[8] O. Loffeld, H. Nies, U. Gebhardt, V. Peters, and S. Knedlik, "Bistatic SAR-Some reflections on Rocca's smile," in Proc. Eur. Conf. Synthetic Aperture Radar, EUSAR, Ulm, Germany, May 2004.

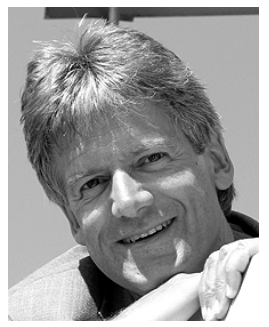

Otmar Loffeld received the Diploma degree in electrical engineering from the Technical University of Aachen, Aachen, Germany, in 1982, the Dr.Eng. degree and the "habilitation' in the field of digital signal processing and estimation theory in 1986 and 1989 , respectively, both from the University of Siegen, Siegen, Germany.

He has worked at the University of Siegen on various problems of optimal filtering, estimation, and control for linear and nonlinear problems. In 1991, he was appointed as a Professor for digital signal processing and estimation theory at the University of Siegen. In 1995, he became a Member of the Center for Sensorsystems (ZESS), which is a central scientific research establishment at the University of Siegen, and has been Vice Chairman there since 2000. Since 2002, he is Speaker of the International Postgraduate Programme Multi Sensorics at the University of Siegen hosted by ZESS. He is author of two textbooks on estimation theory. His current research interests comprise multisensor data fusion, Kalman filtering techniques for data fusion, optimal filtering and process identification, SAR processing and simulation, SAR-interferometry, phase unwrapping, and baseline estimation. A recent field of interest is bistatic SAR processing.

Dr. Loffeld received the scientific award of Nordrhein-Westfalen (Bennigsen-Foerder Preis) in 1990 for his work on applying Kalman filters to phase estimation problems such as Doppler centroid estimation in SAR, phase, and frequency demodulation. He is a member of the Information Technology Society, Association for Electrical, Electronic and Information Technologies (ITG/VDE) and of the IEEE GRSS.

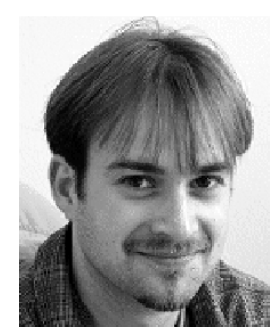

Holger Nies received the Diploma degree in electrical engineering from the University of Siegen, Siegen, Germany, in 1999.

Since 1999, he has been a Member of the Center for Sensorsystems, University of Siegen, and a Lecturer in the Department of Signal Processing and Communication Theory. His current research interests are in the areas of SAR processing, phase unwrapping, and orbit modeling.

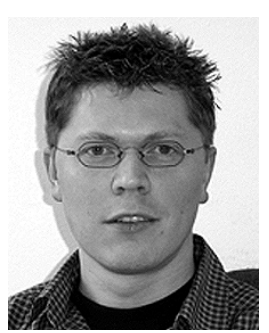

Valerij Peters received the Diploma degree in electrical engineering from the University of Siegen, Siegen, Germany, in 2002.

Since October 2002, he has been a Research Associate at the University of Siegen. His current research interests include mono- and bistatic signal theory, bistatic SAR processing, and computer-based sensor simulation.

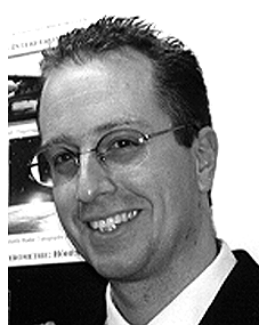

Stefan Knedlik (M'04) received the Diploma degree in electrical engineering and the Dr. Eng. degree in 1998 and 2003, respectively, both from the University of Siegen, Siegen, Germany.

Since 1998, he has been a Member of the Center for Sensorsystems (ZESS), University of Siegen, and a Lecturer in the Department of Signal Processing and Communication Theory. In several projects, e.g., in cooperation with DLR and Dornier, he developed state and parameter estimation strategies within SAR Interferometry. Together with Dr. Otmar Loffeld, he was Principal Investigator for the calibration of the interferometrical baseline for the SRTM/ X-SAR system. Since 2002, he has been Executive Director of the International Postgraduate Programme Multi Sensorics at ZESS. His current research interests include applied estimation theory and data fusion in SAR Interferometry, as well as navigation and communication in sensor webs.

Dr. Knedlik is a member of the German Association of University Professors and University Lecturers (DHV). 